\title{
ANÁlisis FINANCIERO DEL SECTOR AUTOMOTRIZ, UN ESTUDIO APLICADO A LAS EMPRESAS PERTENECIENTES A UNA CIUDAD INTERMEDIA DE UN PAÍS EN VÍAS DE DESARROLLO
} FINANCIAL ANALYSIS OF THE AUTOMOTIVE SECTOR: A STUDY APPLIED TO COMPANIES BASED IN A MEDIUM-SIZED CITY OF A DEVELOPING COUNTRY

Fecha de recepción: 03/12/2018

Fecha de aceptación: 11/05/2020

\section{Resumen}

En el siguiente artículo se presenta un estudio de tipo financiero aplicado a las empresas que pertenecen al sector automotriz en Ecuador y que tienen registrado su domicilio en la ciudad de Cuenca. La finalidad del estudio es la de analizar y comprender cuál fue su desempeño financiero durante el período comprendido desde el año 2010 hasta el 2016, buscando determinar los factores que influyeron en el desempeño de las empresas estudiadas, su comportamiento y las principales características que estas mantuvieron a lo largo del período de estudio. Para este trabajo se manejó un análisis de razones financieras como liquidez, endeudamiento, rendimiento, actividad y valor económico agregado. Los datos utilizados fueron recabados de la Superinten-

\footnotetext{
Universidad del Azuay. E-mail: c.dani_87@hotmail.com

$2 \quad$ Universidad del Azuay. E-mail: chikis9321@gmail.com

$3 \quad$ Universidad del Azuay. E-mail: mapiedra@uazuay.edu.ec
} 
Análisis financiero del sector automotriz, un estudio aplicado a las empresas pertenecientes...

dencia de Compañías y la Asociación de Empresas Automotrices del Ecuador (AEADE). Los resultados obtenidos muestran que el sector automotriz se vio afectado en su desarrollo en el período comprendido entre los años 2010 y 2016, pues el sector destruye su valor económico, ocasionado principalmente por la crisis económica que vivió el país, y de manera específica la ciudad de estudio. El rendimiento de sus activos y patrimonio también disminuyen en dicho período, a pesar de estos resultados el sector logra mantenerse disminuyendo su deuda con terceros, y negociando tiempo de pago con proveedores.

Palabras clave: análisis, razones financieras, liquidez, endeudamiento, rendimiento, sector automotriz, valor económico agregado.

\section{Abstract}

The following article presents a financial study applied to companies of the automotive sector in Ecuador with registered address in the city of Cuenca. The purpose of the study is to analyze and understand their financial performance during the period 2010-2016 in order to determine the factors that influenced the performance, behavior and main characteristics of such companies throughout the study period. For this purpose, an analysis of financial ratios such as liquidity, indebtedness, performance, activity and added economic value was carried out. The data used were collected from the Superintendence of Companies and the Association of Automotive Companies of Ecuador (AEADE). Results show that the automotive sector was affected in its development in the period 20102016, as the sector destroyed its economic value, mainly due to the economic crisis experienced by the country and the city under study in particular. The performance of its assets and equity also decreased in that period. Despite these results, the sector managed to subsist by decreasing its debt with third parties and negotiating payment time with suppliers.

Keywords: analysis, financial ratios, liquidity, indebtedness, performance, automotive sector, economic added value.

JEL: G31, G32, L81, L92. 


\section{Introducción}

En el período comprendido entre los años 2010 al 2016 en el Ecuador se sufrió una serie de cambios económicos a raíz de varias situaciones en el sector externo, como lo fue la crisis económica, lo que llevó a que los organismos que regulan la política financiera ecuatoriana adopten medidas restrictivas frente a productos y procesos que fomentaban la salida de dinero del país. Los principales componentes que caracterizaron esta situación fueron la caída del precio del petróleo y el déficit económico presupuestario que se vivía año a año. Con estas condiciones el Comité de Comercio Exterior (COMEX) decidió reforzar la situación de seguridad que el país debía adoptar, con medidas restrictivas. Una de las áreas afectadas con estas medidas fue sin duda el sector automotriz, y que en este artículo será estudiado hacia el escenario presentado en la ciudad de Cuenca. Este sector de la economía ecuatoriana es de gran importancia por la necesidad de tecnología e investigación que precisa, así como de los ingresos que genera para la sociedad, como son: fuentes de empleo y desarrollo de las empresas productoras de autopartes, entre otras.

Varios de los países que se conocen como en vías de desarrollo de América Latina presentan una alta dependencia del sector primario orientado al mercado internacional, en particular en lo que se refieren a las exportaciones de recursos naturales. Asimismo, estos países presentan una creciente demanda interna de productos importados y una alta dependencia de la demanda de Estados Unidos, su principal destino comercial (Complexity, 2018). Lo expresado anteriormente ha provocado que los países de la región tengan persistentes problemas de déficit externo y que su tasa de crecimiento esté asociada al precio de los commodities que exportan (Alvarado \& Iglesias, 2017). En este contexto el estudio del sector comercial automotriz se convierte en una herramienta muy importante que permitirá describir cuales han sido los principales cambios que han sufrido las empresas comerciales automotrices pertenecientes a dicho sector, domiciliadas en una ciudad categorizada como intermedia de un país conocido como en vías de desarrollo.

En el año 2012 el Comité de Comercio Exterior, como organismo regulador de las políticas públicas nacionales en el ámbito comercial, presentó una resolución cuantitativa, la misma que estuvo impuesta hasta el año 2014, la cual consistía en que a las empresas importadoras de automóviles se les asignaría un límite de cupo de importaciones del cual hacer uso, sea esta en dólares o en cantidades de vehículos importados, dependiendo de manera directa del tamaño de cada empresa que se encontraba en el sector, lo que ocasionó que 
Análisis financiero del sector automotriz, un estudio aplicado a las empresas pertenecientes...

las empresas dedicadas a la comercialización de vehículos presentaran bajas en sus ventas.

El mismo organismo regulador COMEX, a partir del año 2015, elimina la resolución política anteriormente mencionada e incrementa nuevamente el cupo de importaciones a ciertos tipos de vehículos, los cuales debían cumplir con determinadas características definidas previamente (Paspuel, 2015). Sin embargo, este incremento no fue de mayor ayuda, pues toda la economía ecuatoriana se vio afectada con la fuerte recesión económica presentada a mediados del año 2015 (Salgado Defranc, 2016); en este año el país mostró una drástica caída en el PIB debiéndose principalmente a la caída en el precio del petróleo, lo cual terminó afectando a todo el comercio, pues la economía ecuatoriana presentó tan solo un 0,3\% de incremento en su PIB (Editorial, 2016)

En este artículo se analizan datos obtenidos por medio de la Superintendencia de Compañías del Ecuador, en donde se han analizado los estados financieros de las empresas estudiadas e información tomada de la Asociación de Empresas Automotrices del Ecuador (AEADE), buscando obtener hechos específicos que muestren la situación que las empresas automotrices en la ciudad de Cuenca atravesaron durante el período 2010-2016. Algunas de las medidas restrictivas aplicadas en el país fueron el incremento de aranceles y la disminución en cupos de importación (Pico Versoza \& Coello Yagual, 2016).

\section{Marco de antecedentes}

El estudio del componente financiero empresarial es una actividad necesaria en todas las organizaciones, pues implica el análisis de un todo a través del conocimiento de cada uno de sus componentes, examinando la realidad, principios, propiedades y funciones de la organización, obteniendo la información de distintos medios y analizándolos para al fin poder ser un factor de decisiones para la empresa (García Padilla, 2015).

El sector automotriz posee un rol significativo en la economía ecuatoriana, que en los últimos seis años ha presentado una serie de cambios; su desarrollo genera ingresos fiscales para el Estado mediante aranceles e impuestos y crea fuentes de empleo durante los procesos de producción y las actividades relacionadas al comercio del mismo. La eficacia del sector se ha incrementado últimamente y en especial durante los años posteriores a la dolarización cuando la opción de adquirir bienes duraderos fue una alternativa ante la desconfianza de la población en el sistema financiero dado en esa época (Carrillo, 2009). 
Para el año 2010 el gobierno ecuatoriano se encontraba analizando la posibilidad de implementar una restricción cuantitativa a la importación de vehículos para subsanar el déficit comercial. La situación financiera para este sector en el 2010 mejoró considerablemente en relación con el 2009 aumentando en un $18 \%$ sus ventas. En el año 2011 el COMEX comenzó a establecer licencias para la importación de vehículos buscando frenar un $20 \%$ la importación y fomentar el desarrollo nacional del sector, lo que provocó que varias empresas no cumplieran con sus cupos establecidos durante el año, provocando un alza en precios y cambios en la demanda de distintos productos (AEADE, 2010).

En el 2012 el comportamiento del sector se vio mayormente alterado, pues el COMEX mediante la Resolución $\mathrm{N}^{\circ} 66$ estableció restricciones para importar vehículos armados (AEADE, 2012). Para este año existió también una gran restricción en los cupos de importación disminuyendo en un 55\% con respecto al año anterior. Sin embargo, la mejora del poder adquisitivo de los ciudadanos y la disponibilidad de créditos ofertados durante el año 2014 sirvieron de ayuda en la generación de las ventas pues hasta septiembre de ese año se generaron ventas de 86.142 vehículos (Orozco \& Enríquez, 2014).

El año 2015 sin duda fue uno de los años en el que el sector automotriz sufrió varias alteraciones. Entre algunas de las medidas adoptadas durante este período y que afectaron al sector, podemos encontrar el incremento en el arancel de importaciones en un 15\% para los kits de ensamblaje de automóviles, se grabó la importación de tractocamiones con un 5\% de arancel y con un $10 \%$ a los chasises de los kits de ensamblaje a camiones (AEADE, 2015). Los neumáticos también se vieron afectados con la aplicación de salvaguardias del $25 \%$.

El mercado ecuatoriano de vehículos nuevos alcanzó un total de ventas de 63.500 unidades en 2016, según cifras de la Asociación de Empresas Automotrices del Ecuador (AEADE), a partir de lo cual se ha producido una marcada tendencia decreciente, particularmente en los dos últimos períodos, producto de las regulaciones que se definieron para limitar el ingreso de vehículos importados completos o en piezas (Acebo Plaza \& Nuñez, 2017).

Según la Asociación de Concesionarios Automotrices del Ecuador (2014), desde el año 2000, luego del proceso de dolarización en el Ecuador, las ventas de vehículos en el Ecuador mostraron un crecimiento importante y sostenido durante la siguiente década; sin embargo, a partir del año 2012 se produjo un decremento del 13\% y del 6\% del año 2011 al 2012 y del 2012 al 2013 respectivamente, esto es una disminución de 139.893 unidades en el 2011 a 121.446 en el año 2012 y a 113.812 para el 2013. Para el año 2014 se produjo un incremento del 5,5\%, pasando a 120.060 unidades vendidas en la industria automotriz. 
Análisis financiero del sector automotriz, un estudio aplicado a las empresas pertenecientes...

(Valle, Morales \& Fonseca, 2016). El gráfico a continuación muestra de mejor manera las ventas en cada año.

Gráfico 1. Ventas anuales de automóviles del 2010 al 2014

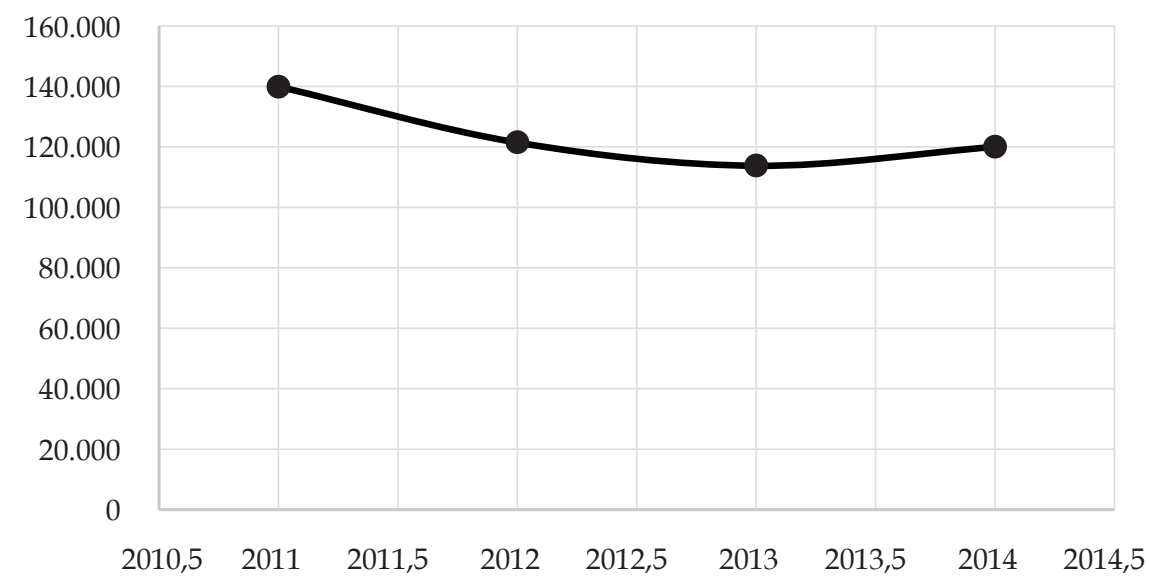

Fuente: elaboración propia.

\section{Procedimientos metodológicos}

Se desarrolla un trabajo de naturaleza cuantitativa, mediante la aplicación de revisiones monetarias e indicadores numéricos los cuales son planteados luego de haber aplicado criterios de búsqueda y recolección de datos para su posterior análisis estadístico. La base para este estudio financiero ha sido el análisis de los distintos indicadores o ratios financieros de liquidez, actividad, rentabilidad y endeudamiento. Dentro de cada uno de estos grupos, se ha seleccionado el o las ratios que más se relacionan con el análisis presentado del sector automotriz. En el desarrollo de este proyecto, las razones financieras permiten identificar la relación entre diferentes años de estudio, con el fin de obtener un resultado de los diversos cambios en el sector, los que permiten evaluar, supervisar y determinar el rendimiento del sector.

- Los indicadores de liquidez miden la capacidad que tiene la empresa para cumplir con sus obligaciones a corto plazo a medida que estas llegan a su vencimiento (Gitman \& Zutter, 2016). 


\section{- Prueba Ácida}

Este indicador financiero muestra la capacidad que mantiene la empresa para cancelar sus obligaciones sin la necesidad de obtener ayuda por parte de los inventarios, por lo que la fórmula se vuelve más exigente.

$$
\text { Prueba Ácida }=\frac{\text { Activo Corriente }- \text { Inventarios }}{\text { Pasivo Corriente }}
$$

- Las razones de actividad miden la eficiencia con la que las empresas utilizan sus activos para el desarrollo de las actividades (Gitman \& Zutter, 2016).

\section{- Cuentas por Pagar}

Este indicador proporciona una medida para conocer cuánto se demora la empresa en pagar a sus proveedores.

$$
\text { Cuentas por Pagar }=\frac{\text { Cuentas por Pagar }}{\text { Promedio diario de compras }}
$$

- Las razones de endeudamiento de una empresa muestran la o las cantidades de dinero de terceras personas que han sido utilizadas para generar utilidades (Gitman \& Zutter, 2016).

\section{- Índice de Endeudamiento}

Este indicador mide los activos totales que han sido financiados por los acreedores de las empresas, es decir cuánta cantidad de dinero de otras personas utiliza la empresa para financiarla.

$$
\text { Índice de Endeudamiento }=\frac{\text { Pasivo } s \text { Totales }}{\text { Activos Totales }}
$$

- Las razones de rentabilidad analizan qué tan bien se generan los retornos de los valores invertidos en la empresa, dando una orientación importante a las personas relacionadas directamente con la empresa (Gitman \& Zutter, 2016). 


\section{- Margen de Utilidad Neta}

Este indicador mide el porcentaje que ha quedado de cada unidad monetaria por las ventas después de retirar todos los costos y gastos, incluyendo los intereses, los impuestos y los dividendos de acciones preferentes.

$$
\text { Margen de Utilidad Neta }=\frac{\text { Utilidad disponible }}{\text { para los Accionistas Comunes }}
$$

\section{- Rentabilidad sobre activos}

El rendimiento sobre activos totales mide la eficacia de la administración para generar utilidades con los activos con los que dispone.

$$
\text { ROA = Margen de utilidad neta * Rotación de Activos }
$$

\section{- Rentabilidad sobre capital invertido}

Esta ratio mide el rendimiento ganado sobre las inversiones que han sido realizadas por los accionistas comunes de la empresa, generalmente a un mayor rendimiento sobre el patrimonio, mejores son los resultados obtenidos para los accionistas.

$$
\text { ROE }=\text { Margen de utilidad neta * Rotación de Activos * Multiplicador de Apalanca- }
$$
miento Financiero

\section{- Valor Económico Agregado (EVA)}

$$
\text { EVA }=\text { UODI - WACC } \times \text { ANDEO }
$$

Conocemos que el EVA es una medida del rendimiento de las empresas que indica cuánto valor se ha creado con la utilización del capital invertido, mismo 
que termina siendo un indicador de gran valor para los accionistas, esta ratio toma en cuenta tres componentes. A continuación se detalla el desarrollo del cálculo de cada uno de los componentes:

UODI. Es la utilidad operativa después de impuestos. Está conformado por la utilidad operativa multiplicada por 1 menos la tasa de impuestos, misma que se encuentra conformada por el 15\% de participación a trabajadores y el impuesto a la renta que se presenta según el año en análisis.

$$
\mathrm{UODI}=\mathrm{EBIT}^{*}(1-\mathrm{t})
$$

ANDEO. Son los activos netos de operación, el cual está integrado por el activo fijo neto (AFN) y las necesidades operativas de financiamiento (NOF).

- AFN. Para obtener este dato se consideró el activo fijo neto de las empresas

- NOF. Este dato se lo obtuvo por medio de las cuentas inventarios, cuentas por cobrar, cuentas por pagar y pasivo a largo plazo las cuales se analizaron en base a la siguiente formula:

$-\mathrm{NOF}=($ Inventarios + Cuentas por cobrar $)-($ Cuentas por pagar + Pasivo a largo plazo)

Luego de obtener estos datos se aplicó la siguiente fórmula para determinar el ANDEO:

$$
\mathrm{ANDEO}=\mathrm{NOF}+\mathrm{AFN}
$$

WACC. Es conocido como el Costo de Capital Promedio Ponderado. Para calcular este porcentaje se empleó la siguiente fórmula:

$$
W A C C=\frac{\text { TOTAL PASIVO }}{\text { TOTAL ACTIVO }} * k d *(1-t)+\frac{\text { PATRIMONIO }}{\text { TOTAL ACTIVO }} * k e
$$

Para obtener el costo de la deuda se debe considerar la tasa de interés al que las empresas obtienen un financiamiento $(\mathrm{kd})$. Es por ello que por el número de empresas que se encentran en análisis se consideró la tasa activa referencial del Banco Central del Ecuador; y para determinar los impuestos se tomó como referencia el porcentaje de impuesto para cada año.

Para obtener el costo de capital (ke) y poder realizar el cálculo del WACC se consideró al ROE, pero debido al número de empresas y la composición 
Análisis financiero del sector automotriz, un estudio aplicado a las empresas pertenecientes...

del patrimonio que presenta cada una de ellas, los datos obtenidos en ciertas empresas eran exagerados, por lo que indicaban cifras demasiado altas o demasiado bajas, distorsionando la información. Para obtenerla mediante un promedio, nos vimos en la necesidad de depurar datos mediante la utilización de cuartiles.

La tabla que se muestra a continuación indica los valores de ke, kd y el impuesto de cada año respectivamente:

Tabla 1. Costos de deuda y patrimonio

\begin{tabular}{ccc}
\hline AÑO & COSTO DE LA DEUDA & COSTO DEL PATRIMONIO \\
\hline 2010 & $9,54 \% *(1-36,25 \%)$ & $34,47 \%$ \\
\hline 2011 & $9,53 \% *(1-35,40 \%)$ & $30,43 \%$ \\
2012 & $9,53 \% *(1-33,55 \%)$ & $21,64 \%$ \\
\hline 2013 & $9,53 \% *(1-33,70 \%)$ & $17,31 \%$ \\
2014 & $9,63 \% *(1-33,70 \%)$ & $16,62 \%$ \\
2015 & $9,76 \% *(1-33,70 \%)$ & $8,87 \%$ \\
\hline 2016 & $9,84 \% *(1-33,70 \%)$ & $6,95 \%$ \\
\hline
\end{tabular}

Fuente: elaboración propia.

La información utilizada como base de este trabajo se obtuvo de la Superintendencia de Compañías, pudiéndose así conseguir una muestra estadística de las empresas del sector automotriz en la ciudad de Cuenca, en el período comprendido entre los años 2010 y 2016. Para conocer cuáles serían las empresas con las que se trabajaría, primero se consideró el ranking de las empresas que presenta la Superintendencia de Compañías, tomando las empresas que se encuentran dentro de la clasificación "Por tamaño", seleccionando las empresas grandes, es decir, las que poseen ingresos superiores a $\$ 5.000 .000$ o tienen más de 200 trabajadores.

Por último, el análisis de las razones financieras se realizó de manera global al sector, estableciendo un promedio general en base al número de empresas con las que se trabajó, considerando el total de las empresas como el peso para la aplicación del promedio, pues en cada año se tiene distintos números de empresas y los resultados de las razones financieras varían de empresa a empresa. Es por ello que con esta aplicación se esperó identificar de manera 
más clara el comportamiento del sector, con el fin de equiparar los resultados para cada año. Cabe anotar que los Estados Financieros son elaborados bajo las Normas Internacionales de Información Financiera.

\section{Análisis de datos}

El análisis de los resultados obtenidos comenzó con un estudio del sector comercial de la ciudad de Cuenca, donde se encontraban tres subclasificaciones, las cuales fueron las siguientes: Sector Comercial al Por Mayor, Sector Comercial al Por Menor y el Sector Automotriz. De este último se obtuvo toda la información para el análisis realizado; se clasificó las empresas mediante el código de Clasificación Industrial Internacional Uniforme (CIIU) correspondiendo para este sector el CIIU 45, que engloba el comercio automotriz en el Ecuador. Durante los años en análisis se obtuvieron datos de distintos números de empresas por año como se muestra en el siguiente cuadro:

Tabla 2. Empresas existentes por año en el sector automotriz cuencano

\begin{tabular}{c|c}
\hline ANOS & EMPRESAS \\
\hline 2010 & 20 \\
\hline 2011 & 21 \\
2012 & 21 \\
\hline 2013 & 21 \\
2014 & 19 \\
2015 & 21 \\
2016 & 14 \\
\hline
\end{tabular}

Fuente: elaboración propia.

De manera general el sector automotriz de Cuenca ha crecido durante los últimos seis años en análisis (2010-2016). Los años que presentan disminuciones son 2010, 2013 y 2015 como resultado de distintas acciones políticas que modificaron la tendencia del sector. Para el análisis realizado, se tomó en consideración la Prueba Ácida, las Cuentas por Pagar, el Índice de Endeudamiento, Margen de Utilidad Neta, ROA, ROE y por último el Valor Económico Agregado (EVA); los datos obtenidos del sector se resumen en la siguiente tabla: 
Análisis financiero del sector automotriz, un estudio aplicado a las empresas pertenecientes...

Tabla 3. Resultados de indicadores financieros aplicados

\begin{tabular}{cccccccc}
\hline AÑOS & $\begin{array}{c}\text { PRUEBA } \\
\text { ÁCIDA }\end{array}$ & $\begin{array}{c}\text { CUENTAS } \\
\text { POR PAGAR }\end{array}$ & $\begin{array}{c}\text { ÍNDICE DE } \\
\text { ENDEUDA- } \\
\text { MIENTO }\end{array}$ & M.U.N & ROA & ROE & EVA \\
\hline 2010 & 0,70 & 63 & $72,49 \%$ & $4,00 \%$ & $7,99 \%$ & $29,03 \%$ & $\$(188.753,10)$ \\
\hline 2011 & 0,87 & 66 & $64,35 \%$ & $4,52 \%$ & $8,81 \%$ & $24,72 \%$ & $\$ 123.675,38$ \\
\hline 2012 & 0,85 & 74 & $64,75 \%$ & $4,62 \%$ & $7,67 \%$ & $21,76 \%$ & $\$ 113.843,94$ \\
\hline 2013 & 0,77 & 76 & $64,04 \%$ & $4,44 \%$ & $6,20 \%$ & $17,25 \%$ & $\$ 7.081,32$ \\
\hline 2014 & 0,96 & 80 & $63,89 \%$ & $3,72 \%$ & $6,01 \%$ & $16,64 \%$ & $\$ 42.786,67$ \\
\hline 2015 & 0,80 & 84 & $59,97 \%$ & $3,08 \%$ & $3,36 \%$ & $8,40 \%$ & $\$(433.755,42)$ \\
\hline 2016 & 1,25 & 102 & $52,97 \%$ & $3,42 \%$ & $3,99 \%$ & $8,49 \%$ & $\$ 80.053,51$ \\
\hline
\end{tabular}

Fuente: elaboración propia.

La prueba ácida del sector comercial automotriz presenta valores eficientes para el mercado, salvo los años 2010 y 2013 que presentaron 0.70 y 0.77 respectivamente, lo que denota que las empresas pudieron incumplir con algunos pagos. Para el año 2016 es notable el incremento y mejora de la situación al presentar un resultado de 1.25 lo que daba estabilidad y confianza a las empresas para cubrir sus pasivos.

Gráfico 2. Prueba Ácida

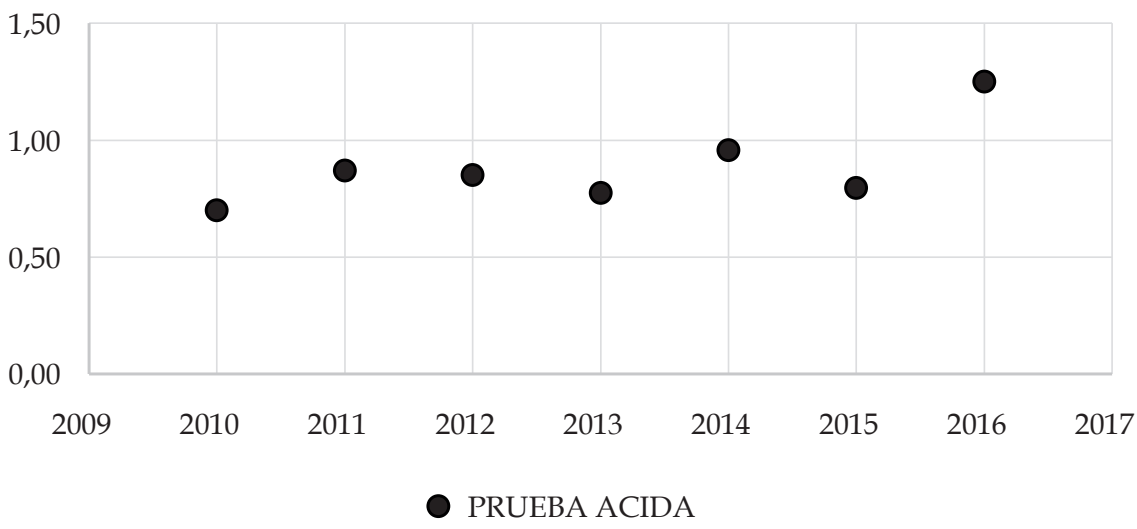

Fuente: elaboración propia. 
Las cuentas por pagar presentaron una mejora notable año con año pues de un período de pago de 63 veces en el 2010 aumentaron a 102 veces en el 2016, aumentando en 10 puntos cada dos años. Estos valores que reflejan una mejora del sector se deben al notable poder de negociación que se pudo tener con los proveedores, o bien a la aplicación de algunas políticas gubernamentales aplicadas que facilitaron esta situación.

Gráfico 3. Cuentas por pagar

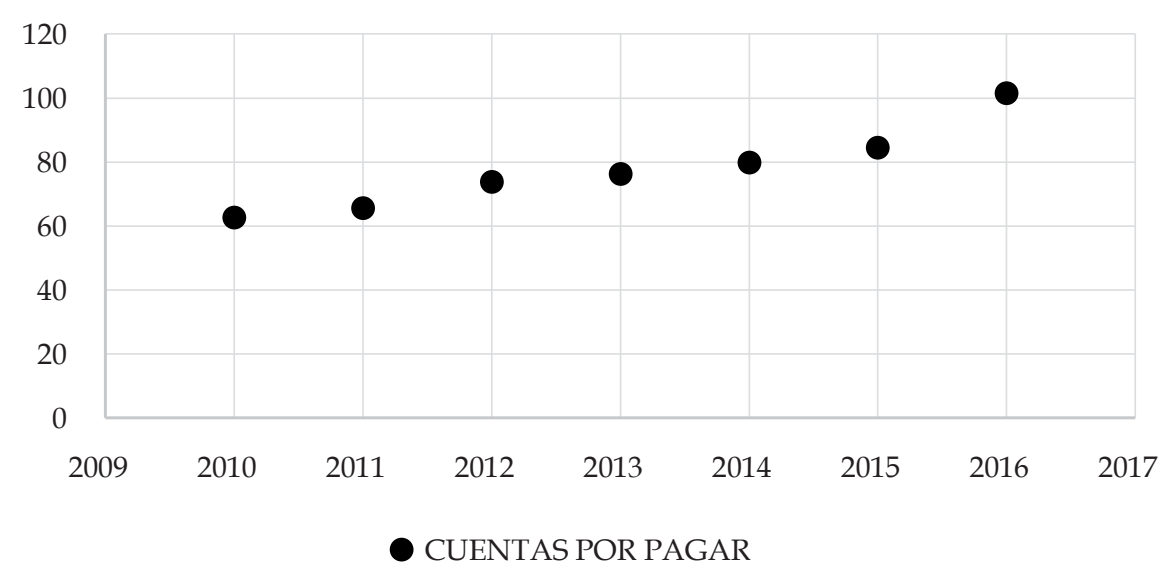

Fuente: elaboración propia.

El índice de endeudamiento del sector automotriz ha disminuido de un año a otro dentro del período analizado, comenzando en el 2010 con un 72,49\%, mostrando a las empresas de un sector automotriz bastante endeudadas, hasta el año 2016 con un porcentaje de 52,97\%, que muestra empresas de un sector automotriz en óptimas condiciones de trabajar con su propio capital y dejar de ser financiado por terceros, situación que se considera la mejor si tomamos como punto importante la situación económica que atraviesa el país. 
Análisis financiero del sector automotriz, un estudio aplicado a las empresas pertenecientes...

Gráfico 4: Índice de Endeudamiento

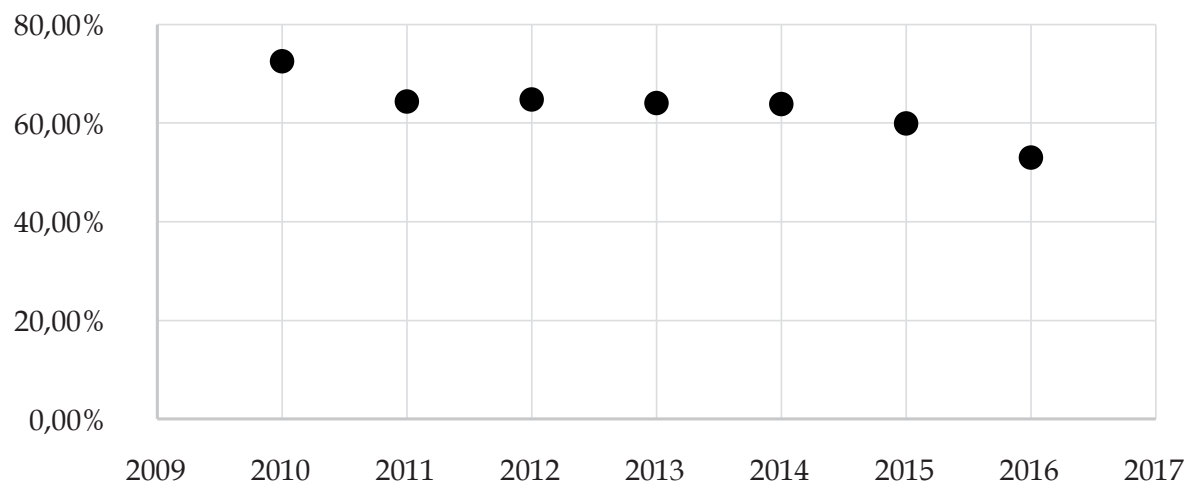

Fuente: elaboración propia.

El margen de utilidad neta del sector automotriz en los años de análisis presentados ha ido disminuyendo con cada período de tiempo analizado empezando en al año 2010 con un $4 \%$ hasta el 2016 con un 3,42\%, con una variación de $0,58 \%$ puntos de diferencia. El margen de utilidad mostrada es baja para un sector que necesita de tecnología de punta para su desenvolvimiento; sin embargo, también es cierto que la ciudad analizada no es tan grande, entonces los valores estarán en relación a estas variables de mercado para ser analizados, lo que se podría considerar como valores normales o en concordancia al sector (ciudad) analizado.

Gráfico 5. Margen de Utilidad Neta (MUN)

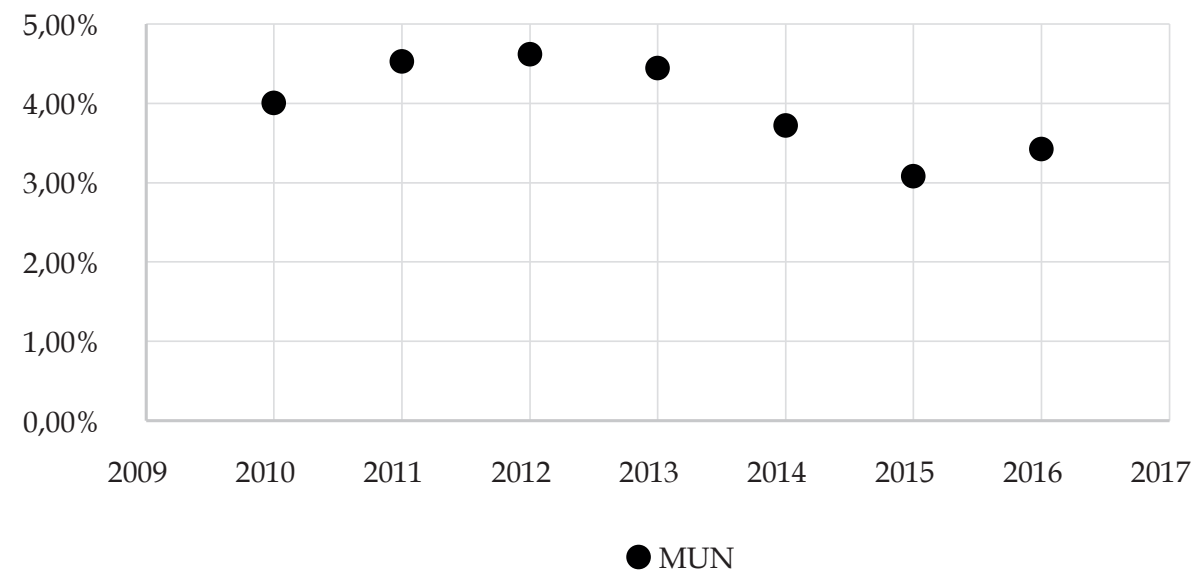

Fuente: elaboración propia. 
El rendimiento sobre activos disminuye notablemente año con año. En el 2010 presenta un ROA de 7,99\%, el 2014 muestra un 6,01\% y finalmente el año 2016 muestra un 3,99\%. Estos valores dan a conocer claramente el problema de las empresas de este sector para generar beneficios, problemas marcados en parte por las políticas implantadas al sector comercial general del cual es parte el sector automotriz, disminuyendo en casi $5 \%$ entre el año inicial y el final. Si bien fueron bajos los valores mostrados, ninguno fue negativo, lo que representa ganancia efectiva en el uso de sus activos disponibles.

Gráfico 6. Rendimiento sobre Activo (ROA)

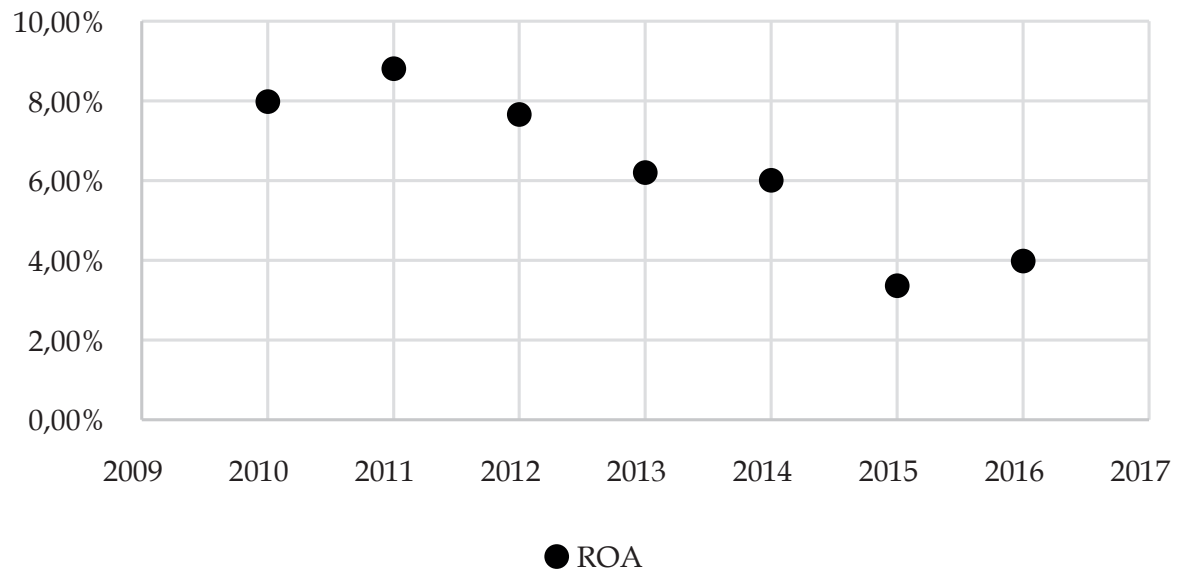

Fuente: elaboración propia.

El rendimiento sobre el patrimonio disminuye aceleradamente desde el 2010 , con un porcentaje de $29,03 \%$ de ganancia para los accionistas del sector, hasta un $8,49 \%$ en el 2016. Los valores se mantienen en un rango del $20 \%$ hasta el 2013, año a partir del cual comienzan a decrecer con una diferencia de 20 puntos desde el $2010 \mathrm{al}$ 2016. Los accionistas no recibieron beneficios mayores como en los años iniciales, pues tampoco se pudo aportar en igual manera. La crisis económica que se empezó a sentir a partir del 2013 puede ser causa principal de estas variaciones. 
Análisis financiero del sector automotriz, un estudio aplicado a las empresas pertenecientes...

Gráfico 7: Rendimiento sobre Capital (ROE)

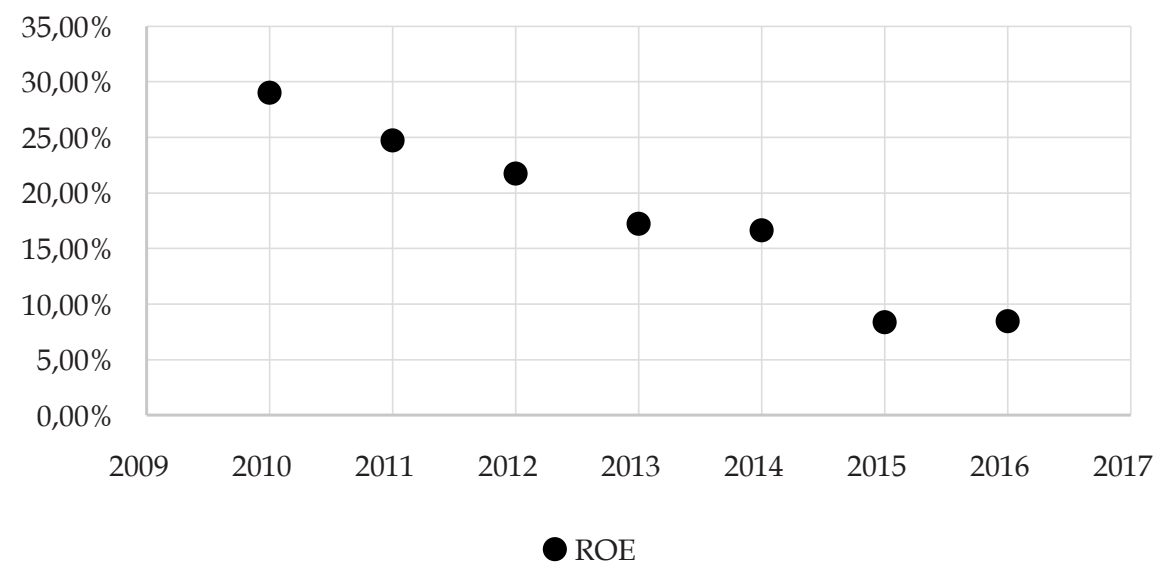

Fuente: elaboración propia.

El valor económico agregado del sector automotriz en la ciudad de Cuenca muestra cifras que destruyen valores en los años que desde un inicio mostraron variaciones en todo el análisis como son 2010, 2013 y 2015. Estos valores hablan de un sector automotriz afectado por la crisis económica andina y mundial, más los distintos reglamentos gubernamentales para afrontarlas de las cuales a inicio del último año se han podido ir recuperando. A partir del $2011 \mathrm{el}$ sector mostró altibajos que desencadenan con la cifra presentada en el 2015 de $\$ 433.755,21$ de pérdidas para el sector, volviendo en el 2016 a recuperar el valor económico sectorial.

Gráfico 8. Valor Económico Agregado (EVA)

EVA

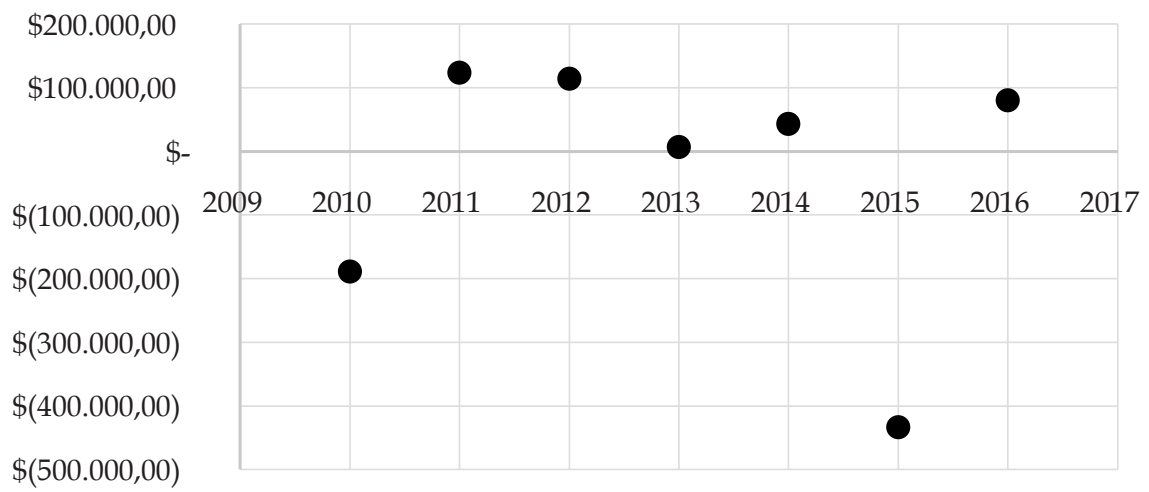

Fuente: elaboración propia. 


\section{Consideraciones finales}

En el estudio financiero realizado al sector comercial automotriz ubicado en la ciudad de Cuenca durante el período 2010 al 2016 se pudo comprobar en aspectos generales la importancia de este componente para el comercio ecuatoriano. Aunque no fue una buena etapa por la situación económica que atraviesa el Ecuador, fue importante analizar su comportamiento durante este período. La crisis económica mundial afectó directamente al país y a la ciudad de Cuenca. Esto fue posible notarlo en el cálculo del Valor Económico Agregado (EVA), donde los sectores en los años 2010 y 2015 perdieron valor con $\$ 188.753,10$ y $\$ 433.755,42$ respectivamente. Esto responde a factores tales como la administración económica y las soluciones que buscó el Ecuador para poder sobrellevar la crisis, tomando como medidas las restricciones a los cupos de importación, frenando en gran medida el desarrollo de este sector.

Los indicadores económicos analizados en este artículo revelan un desempeño favorable para el sector automotriz en la ciudad de Cuenca, desde el punto de vista que las empresas empezaron a tomar medidas de seguridad en su patrimonio para no verse afectadas por una posible falta de liquidez debido a los decretos establecidos para enfrentar la crisis. Varias firmas ante la situación que atravesaba el país presentaron una disminución en ventas y mercadería, lo que ocasionó en varios casos despidos de personal, todo esto no por lograr altos números de rendimiento, sino suficientes para mantenerse en medio de la situación económica del país.

El índice de endeudamiento disminuyó, las empresas durante los períodos más difíciles cuidaban el capital y los activos con los que contaban, preservando su estabilidad y tranquilidad económica, al momento de afrontar las políticas aplicadas y con impacto directo en la importación de vehículos, debido a que se empezó a ensamblar marcas internacionales en el país. Sin embargo, las empresas por trimestre perdían con esta política la posibilidad de adquirir entre 1000 a 2000 vehículos, generando un factor negativo para estos comerciantes, pues no podían cubrir con las exigencias del cliente. Ahora a las empresas les queda esperar la eliminación total o parcial de las restricciones impuestas, y nuevamente analizar el desempeño de los sectores económicos, sobre todo del sector automotriz.

Con el tiempo un estudio sectorial tal como este será imprescindible para contar con una nueva radiografía del sector; con el paso de los años se verá cómo fue su evolución, los nuevos retos y las nuevas oportunidades, pues es un sector que fomenta el desarrollo de varios sectores a estos ligados y, por lo tanto, es visto como uno de los principales aportes al desarrollo de un país. 
Análisis financiero del sector automotriz, un estudio aplicado a las empresas pertenecientes...

\section{Referencias bibliográficas}

Acebo Plaza, M., \& Nuñez, A. (2017). Estudios industriales, orientación estratégica para la toma de decisiones. Guayaquil. Recuperado de: http://www. espae.espol.edu.ec/publicaciones

Asociación de Empresas Automotrices del Ecuador (AEADE) (2010). Anuario 2010. Recuperado de: http:/ / aeade.net/wp-content/uploads/2016/11/ ANUARIO-2010.pdf

Asociación de Empresas Automotrices del Ecuador (AEADE) (2012). Anuario 2012. Recuperado de: http:/ / aeade.net/wp-content/uploads/2016/11/ ANUARIO-2012.pdf

Asociación de Empresas Automotrices del Ecuador (AEADE) (2013). Anuario 2013 Recuperado de: http:/ / aeade.net/wp-content/uploads/2016/11/ ANUARIO-2013.pdf

Asociación de Empresas Automotrices del Ecuador (AEADE) (2015). Anuario 2015 Recuperado de: http:/ / aeade.net/wp-content/uploads/2016/11/ ANUARIO-2015.pdf

Alvarado, R., \& Iglesias, S. (2017). Sector externo, restricciones y crecimiento económico en ecuador. Problemas del Desarrollo, 83-106.

Carrillo, D. (2009). Diagnóstico de Sector Automotriz. Instituto Nacional de Estadísticas y Censos. Recuperado de: http://www.uasb.edu.ec/UserFiles/381/File/AUTOMOTRIZ.pdf

Complexity, T. O. (2018). Complexity, The Observatory of Economic. Recuperado de: https:/ / atlas.media.mit.edu/es /

El Comercio (2016). Caída del crudo y sus efectos. El Comercio. Recuperado de: https://www.elcomercio.com/opinion/editorial/opinion-caida-crudo-efectos.html

García Padilla, V. M. (2015). Análisis Financiero. Un Enfoque Integral. México: PATRIA S.A.

Gitman, L., \& Zutter, C. (2016). Principios de Administración Financiera. México: Pearson.

Orozco, M., \& Enríquez, C. (2014). Las ventas del sector automotor, estancadas. EL COMERCIO. Recuperado de: https://www.elcomercio.com/actualidad/ventas-sector-automotor-estancadas-ecuador.html

Paspuel, W. (2015). El Comex incrementó el cupo para importar vehículos. EL COMERCIO. Recuperado de: https://www.elcomercio.com/actualidad/ comex-incremento-cupo-importacion-vehiculos.html 
Pico Versoza, L. M., \& Coello Yagual, R. R. (2016). Análisis de la restricción a las importaciones del sector automotriz en el Ecuador periodo 2010 - 2015 y su impacto en la economía. INNOVA, 128 -142.

Salgado Defranc, J. (2016). El PIB no creció el 2015. EL COMERCIO. Recuperado de: https:/ / www.elcomercio.com/cartas/pib-crecimiento2015-economia-ecuador-recesion.html

Valle, A., Morales, L., \& Fonseca, Z. (2016). ESTRATEGIA ORGANIZACIONAL Y LA RENTABILIDAD EN EMPRESAS DEL SECTOR AUTOMOTRIZ DE LA ZONA CENTRAL DEL ECUADOR. Recuperado de: https:/ / dialnet. unirioja.es/servlet/articulo?codigo $=5767779$

(C) 2020 por los autores; licencia otorgada a la revista Escritos Contables y de Administración. Este artículo es de acceso abierto y distribuido bajo los términos y condiciones de una licencia Atribución-No Comercial 4.0 Internacional (CC BY-NC 4.0) de Creative Commons. Para ver una copia de esta licencia, visite https://creativecommons.org/licenses/by-nc/4.0/ 